\title{
ERRATA, VOLUME 10
}

R. E. Chamberlin, $A$ class of unknotted curves in 3-space, pp. 149157.

Professor R. H. Bing has given an example showing the step at the beginning of the second paragraph on page 157 is incorrect. The theorem on page 150 which depends on this step is also incorrect as shown by an example of Dr. L. K. Barrett. Additional hypotheses can be added to give a correct theorem, but none that the author has investigated seem to give a theorem of much interest.

Jack Segal, Hyperspaces of the inverse limit space, pp. 706-709.

Page 708, line 2 from the bottom. For “dim $C\left(X_{i}\right) \leqq k "$ read "dim $C\left(X_{i}\right) \leqq k<\infty$."

Page 709. Theorem 2.1 should read "If $X$ has property $k$, then $\operatorname{dim} C(X) \leqq k$. (The proof is trivial.)"

Shin-ichi Izumi, Two theorems concerning convergence of Fourier series, pp. 744-754.

Page 749. Replace line 10 by

$$
\text { “ }|J| \leqq\left|\int_{-\pi}^{\pi}[\phi(u+h)-\phi(u)] d u \int_{|u|}^{\pi} L_{n}(t) d t\right|+o(h) . "
$$

Page 749, line 12. Replace " $=\mathrm{o}(h)$ " by " $+o(h)=o(h) . "$

The author expresses his hearty thanks to Mr. Chen Yung-Ming who has informed him of this error.

E. H. Rothe, $A$ note on gradient mappings, pp. 931-935.

Page 932. The following assumption should be added to those contained in the statement of Theorem 1.1:

"Under the map $D(x, h): V \rightarrow E^{*}$, the image of a bounded subset of $V$ is bounded."

I am indebted to Professor T. Ando of Hokkaido University for bringing to my attention the omission of this hypothesis actually used in the proof of Theorem 1.1.

\section{ERRATA, VOLUME 11}

L. Carlitz, A theorem on permutations in a finite field, pp. 456-459.

It is easily seen that hypothesis (3) of the theorem implies that the polynomial must be a permutation polynomial. 
Page 458, line 12. Replace formula (15) by

$$
(-1)^{s} C_{m, s} b_{s}^{(r)}=(-1)^{r} C_{m, r} b_{s}^{(r)} \quad(0 \leqq r \leqq m ; 0 \leqq s \leqq 2 m) .
$$

Page 458 , last line. Replace $<$ by $\leqq$.

Page 459, line 4. Replace by $k=1+\cdots+p^{t}(0<t<n)$. 